\title{
Dissociation of processing time and awareness by the inattentional blindness paradigm ${ }^{\text {is }}$
}

\author{
Shih-Yu Lo, Su-Ling Yeh* \\ Department of Psychology, National Taiwan University, No. 1, Sec. 4, Roosevelt Road, Taipei 106, Taiwan
}

\section{A R T I C L E I N F O}

\section{Article history:}

Received 11 November 2007

Available online 7 May 2008

\section{Keywords:}

Awareness

Consciousness

Inattentional blindness

Processing time

Texture segregation

The Simon effect

\begin{abstract}
A B S T R A C T
Consciousness researchers are interested in distinguishing between mental activity that occurs with and without awareness (i.e., explicit versus implicit processes). The inattentional blindness (IB) paradigm is an excellent tool for this question because it permits the independent manipulation of processing time and awareness. In the present study, we show that implicit texture segregation can occur during inattentional blindness, provided that the texture is exposed for a sufficient duration. In contrast, a Simon effect does not occur during inattentional blindness, even with similar exposure duration of the critical stimulus. This reveals a dissociation between processing time and awareness, consistent with the possibility of separate processing streams for explicit and implicit visual perception.
\end{abstract}

(C) 2008 Elsevier Inc. All rights reserved.

\section{Introduction}

Perception without visual awareness (i.e., implicit processing) is an important issue in the fields of vision and attention because it fills the gap between what has been processed and what has been seen. However, temporal properties of these implicit processes are seldom examined, due to methodological difficulties in teasing apart the influences of processing time and awareness, and a prevalent assumption that long processing time unavoidably leads to the involvement of awareness. In the present study, we attempt to overcome the methodological difficulties by adopting an appropriate paradigm and thus are able to examine whether separate processing streams are responsible for implicit and explicit visual perception.

Classical evidence for perception without awareness comes from neuropsychological patients, as in cases of blindsight (Danckert \& Rossetti, 2005; Stoerig \& Cowey, 1997; Weiskrantz, 1986), neglect, amnesia, and agnosia (see Faulkner \& Foster, 2002, for a review). These studies, while compelling, leave questions unanswered about perception without awareness in the healthy brain. To address this question, several experimental paradigms have been designed to probe mental processes without awareness in healthy individuals (see Kim \& Blake, 2005, for a review). These paradigms can be generally divided into two major classes. One renders stimuli invisible by inducing competition in the temporal domain, typically by having the critical stimulus closely preceded or followed by another stimulus (usually within $100 \mathrm{~ms}$ ) so that the processing of the critical stimulus is disrupted. Metacontrast masking (e.g., Enns \& Di Lollo, 1997), the attentional blink (e.g., Raymond, Shapiro, \& Arnell, 1992), and repetition blindness (e.g., Kanwisher, 1987) are all examples of procedures in this class.

A second class of procedures involves inducing competition between two stimuli in the spatial domain. For example, in bistable figures such as the vase-face pattern (Rubin, 1921), one of the two figures is perceived to appear and disappear alternately with the other one, permitting only one perceptual state to exist at any given time. In binocular rivalry, only one of the

\footnotetext{
This research was supported by the National Science Council of Taiwan (NSC93-2413-H-002-017 and NSC94-2752-H-002-008-PAE).

* Corresponding author. Fax: +88622362 9909.

E-mail address: suling@ntu.edu.tw (S.-L. Yeh).
} 
two percepts can enter into awareness at any given time (Wade \& Wenderoth, 1978; White, Petry, Riggs, \& Miller, 1978). In motion-induced blindness (MIB, Bonneh, Cooperman, \& Sagi, 2001; Carter \& Pettigrew, 2003; Graf, Adams, \& Lages, 2002), a salient static visual stimulus becomes invisible when it is presented simultaneously with moving dots for some time (Hsu, Yeh, \& Kramer, 2004, 2006; Mitroff \& Scholl, 2005; Montaser-Kouhsari, Moradi, Zandvakili, \& Esteky, 2004). In perceptual filling-in (PFI, Ramachandran \& Gregory, 1991; Ramachandran, Gregory, \& Aiken, 1993; Spillmann \& Kurtenbach, 1992; Welchman \& Harris, 2000, 2001), prolonged viewing of the central fixation causes a peripherally presented gray patch to be gradually replaced in awareness by the surrounding moving dots. Finally, in Troxler fading (Troxler, 1804) a peripheral stimulus disappears after prolonged fixation (Clarke, 1957, 1960, 1961; Clarke \& Belcher, 1962). A common feature of all these examples of spatially induced competition is that the perceptual states observed are intermittent rather than constant, appearing and reappearing in alternation, and varying with each observer. This makes it difficult to control the duration for a given percept.

For the class of methods operating in the temporal domain to induce perception without awareness, the necessarily brief presentation time of each stimulus leads to a temporal constraint on experimentation. For the method involving spatial competition, it is the involuntary oscillation between two perceptual states that causes the temporal constraint. As such, both types of procedures make it difficult to disentangle the possibly separate roles played by processing time and the emergence into awareness of a stimulus in perception.

In addition to methodological difficulties, there is another reason why few studies have examined the relationship between processing time and awareness; that is, an implicit assumption that awareness co-varies with processing time: with more time available for processing, it is more likely for the stimulus to reach awareness. For example, Destrebecqz and Cleeremans (2001) manipulated the response-stimulus temporal interval and found two qualitatively different learning processes: implicit learning occurs when no response-stimulus interval is provided whereas explicit learning occurs when a 250-ms interval is provided. The interdependence of processing time and awareness is consistent with the view that there is one stream of information processing.

This single-stream view is also embedded in early information processing theories. For example, in the classic debate of early (Broadbent, 1958) versus late (Deutsch \& Deutsch, 1963) selection, information is assumed to flow within one stream, and the debate focuses on the locus of attentional selection. However, recent studies have hinted at the possibility that there may be more than one stream of information processing (e.g. Milner \& Goodale, 1995; Ungerleider \& Mishkin, 1982). For example, in the neuronal global workspace model of consciousness (Dehaene \& Naccache, 2001) there are two neural systems in the brain: one contains independent modules (e.g., areas V1, V4, and MT, etc.) dealing with different types of incoming stimuli (e.g., line segments, color signals, and motion signals, etc.), and the other is the global workspace responsible for integrating information from these modules. The first system processes information automatically and implicitly, while the second system operates as a gate to filter the incoming information up to the consciousness level.

Given the possibility that implicit and explicit processing may be separated into two streams, it appears that long processing time does not necessarily lead to the involvement of awareness. To demonstrate this, we need a method that can render stimuli inaccessible to visual awareness, but with fewer temporal constraints. For this purpose, the inattentional blindness paradigm (IB, Mack \& Rock, 1998; Rock, Linnet, Grant, \& Mack, 1992) is an excellent tool. In this paradigm, participants are asked to conduct a certain task, and the stimulus of interest is presented in the background. Under such an experimental setting, most participants cannot report the presence of the background pattern, and thus the background pattern can be regarded as being outside of awareness. The IB paradigm has two advantages as to the independent manipulation of processing time and awareness: (1) it does not require a very brief exposure duration, and thus a wider range of processing time of a given process can be probed, and (2) observers can be continuously unaware of the critical stimulus in this paradigm, unlike MIB or PFI, thus keeping a given perceptual state relatively constant.

In the current study, we used the IB paradigm and demonstrated a double dissociation between processing time and awareness. For some process (i.e., texture segregation), its occurrence is determined by processing time but not awareness (Experiment 1), while for another process (i.e., the Simon effect) its occurrence is determined by awareness but not processing time (Experiment 2). This dissociation supports the view of separate streams for explicit and implicit information processing.

\section{Experiment 1}

The IB paradigm was originally designed by Rock, Mack, and their colleagues (Mack \& Rock, 1998; Mack, Tang, Tuma, Kahn, \& Rock, 1992; Rock et al., 1992). In one of their studies, Mack and Rock (1998) asked participants to compare the length of the vertical and horizontal lines of a large cross, which was superimposed on a background field. After several trials containing homogenous backgrounds, one quadrant of the background field changed its texture. Upon completion of this critical trial the experimenter asked the participants whether they had seen any change in the background, and their mere chancelevel performance led to the conclusion that attention is necessary for texture segregation. Mack and Rock (1998) called the inability to report the unexpected stimulus inattentional blindness (IB), and the stimulus of interest (although usually in the background) the critical stimulus.

However, failure to report the background may be due to observers' inability to memorize it (inattentional amnesia, Wolfe, 1999) rather than being blind to the unattended background. To provide a more sensitive measure, Moore and Egeth 
(1997) modified this paradigm and used an online measure of the unattended background, which contained the inducing portion of the Ponzo (railroad) illusion (i.e., the upright or inverted V shape of the railroad track). The participants were asked to judge which of the two horizontal lines in the foreground (similar to those in Fig. 2 A) was longer. Although the participants could not report having seen the $\mathrm{V}$ shape in the background, their performance of this line-length judgment was nevertheless biased by it. This finding indicates that even when observers cannot explicitly report seeing the background pattern, some processing is still taking place.

Moore and her colleagues further provided an operational definition for the term inattention: when participants deny seeing the critical stimulus and their forced-choice responses to it are at chance level (Moore, Grosjean, \& Lleras, 2003; Moore, Lleras, Grosjean, \& Marrara, 2004). However, we think the term unawareness better captures the essence of what has been probed in this paradigm. Inattention is an inferred status but unawareness can be confirmed when observers deny seeing the critical stimulus and this subjective report is confirmed by the objective measure of forced-choice response. As well, the use of awareness rather than attention is more conservative, if attention is considered to be a gate to awareness or consciousness (Dehaene \& Naccache, 2001).

In this experiment we used the modified IB paradigm of Moore and colleagues to test whether texture segregation occurs without awareness. We aimed at using texture segregation as the candidate process in order to examine the possible dissociation of awareness and processing time, in contrast with the results of another process (i.e., the Simon effect) in Experiment 2. The exposure duration of the critical stimulus was 200 or 500 ms, manipulated as a between-subject factor.

\subsection{Participants}

Two groups of undergraduates at the National Taiwan University $(N=44$ in the 200 - $\mathrm{ms}$ exposure session and $N=46$ in the 500-ms exposure session) participated in the experiment individually to gain course credits. All were naïve about the purpose of the study.

\subsection{Stimuli and design}

Stimuli were displayed on a 21-inch calibrated EIZO FlexScan T966 color monitor (refresh rate $=100 \mathrm{~Hz}$ ) that was controlled by an IBM compatible personal computer with a Visual Stimulus Generator card (VSG2/3F, Cambridge Research System). The participant sat in a dark room, with his or her head positioned on a chin-rest at a viewing distance of $100 \mathrm{~cm}$.

Each trial was comprised of three displays: the fixation, target, and mask displays (Fig. 1). The fixation display contained a white cross $\left(0.54^{\circ} \times 0.54^{\circ}\right.$ in width and length) on a gray background $\left(55 \mathrm{~cd} / \mathrm{m}^{2}\right)$. The target display contained two horizontal white lines $\left(86 \mathrm{~cd} / \mathrm{m}^{2}\right.$, with a vertical distance of $\left.3^{\circ}\right)$ and a background field, which consisted of 253 Gabor patches $\left(11.8^{\circ} \times 11.8^{\circ}\right.$ in an $11 \times 23$ matrix). One single Gabor is defined as: $G(x, y)=\mathrm{e}^{-\left(x^{2}+y^{2}\right) / 2 \sigma^{2}} * \cos [2 \pi *(x * \cos \theta+y * \sin \theta) / p]$, where $\theta$ controls the orientation, and $p$ denotes the period of the modulating sinusoid, which was fixed at $0.17^{\circ}$. The $\sigma$ value of the Gaussian was $0.1^{\circ}$. The mask display was comprised of black-and-white random dots.

Two kinds of trials (pattern-matrix versus random-matrix) were used in which the target display differed in the background field. The pattern-matrix trial (Fig. 2A) consisted of horizontal and vertical Gabors, oriented in such a way that the texture boundaries formed the inducing track (an upright or an inverted V shape) as in the Ponzo illusion. Gabors in the random-matrix trial (Fig. 2B) were oriented randomly within the $11 \times 23$ matrix. The two horizontal lines in a pattern-matrix trial were of equal length $\left(3.6^{\circ} \mathrm{long}\right)$, whereas in a random-matrix trial they were always of different lengths, which were chosen randomly from one of the four following pairs: $\left(3.2^{\circ}, 3.4^{\circ}\right),\left(3.4^{\circ}, 3.6^{\circ}\right),\left(3.6^{\circ}, 3.8^{\circ}\right), a^{\circ}{ }^{\circ}\left(3.8^{\circ}, 4^{\circ} 1^{\circ}\right)$. The length differences between the two horizontal lines were around $0.2^{\circ}$, the same as used in Moore and Egeth (1997).

Each trial began with the fixation display, presented for $1000 \mathrm{~ms}$, with an $800 \mathrm{~Hz}$ tone as a warning signal for the last $200 \mathrm{~ms}$. The target display was then presented for either 200 or $500 \mathrm{~ms}$, followed by a mask display which remained on the screen until the experimenter initiated the next trial. Participants were instructed to perform a line-length judgment task, by reporting orally either "top" or "bottom" (forced choice) to indicate which of the two horizontal lines was longer, and the experimenter recorded the responses.

\subsection{Procedure}

Trials were divided into three blocks in the following order: practice, pre-inquiry, and inquiry (Fig. 3). The practice block contained 10 random-matrix trials. The pre-inquiry block contained 16 pattern-matrix trials (half with upright V and half inverted V) and 16 random-matrix trials, presented in a randomized order. The inquiry block contained eight trials, in which the 4th, 7th, and 8th trials were pattern-matrix trials, called, respectively, inattention (IA), divided attention (DA), and focal attention (FA) trials, and the rest were random-matrix trials. In the IA and DA trials, the orientation of the $V$ shape was counterbalanced between participants, and after the line-length judgment task, the participants were asked the following three questions successively. (1) Direct query: "Did you notice any pattern in the background of the last trial?" (yes/no). (2) V shape discrimination: "If the background pattern was a V shape, was it an upright V or an inverted V?" While asking this question, the experimenter presented a card with an upright $\mathrm{V}$ and an inverted $\mathrm{V}$, asking participants to point to one of them (two-alternative forced choice). (3) Confidence rating: "How sure do you feel about your last answer?" At this time, the participants were shown a 3-point rating scale (1. not sure at all, 2. somewhat sure, and 3. very sure), and were asked to indicate 


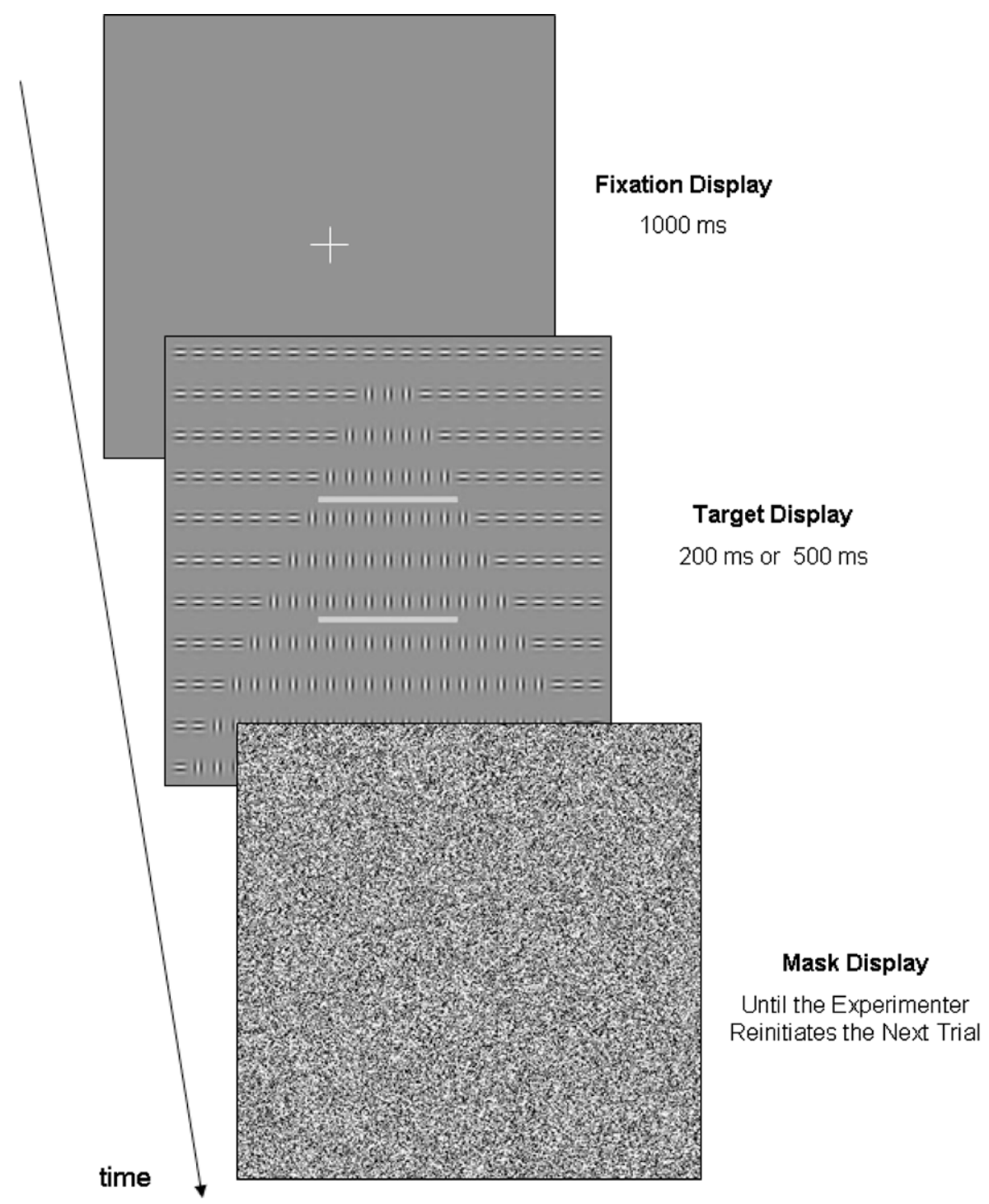

Fig. 1. Stimuli presented in a single trial in Experiment 1.

the most appropriate one. In the final (FA) trial, the participants were asked to attend to the background only, conduct the V shape discrimination, and rate their confidence of the shape discrimination. At the end of the experiment, the participants were asked: "Before the first question in trial 36, did you see any pattern in the background?" This question was meant to double check that the participant had not noticed, in the pre-inquiry block, that there was a pattern in the background. A positive answer to this question would lead to the exclusion of the participant's data.

\subsection{Results}

Data from one and five participants were excluded in the 200- and 500-ms duration sessions, respectively. Chance level (50\%) was served as the baseline for statistical testing. The accuracy of V shape discrimination was $60 \%\left(\chi^{2}=1.88, p>.05\right)$, $44 \%\left(\chi^{2}=0.58, p>.05\right)$, and $91 \%\left(\chi^{2}=28.49, p<.05\right)$ for IA, DA, and FA trials, respectively, in the 200-ms session; and it was $51 \%\left(\chi^{2}=0.02, p>.05\right), 63 \%\left(\chi^{2}=2.95, p>.05\right)$, and $100 \%\left(\chi^{2}=41, p<.05\right)$ in the 500 -ms session. In both sessions, the accuracy in the IA trials was not significantly higher than chance level, thus confirming that the participants were indeed unaware of the background pattern.

The formation of texture segregation was inferred from the results of the line-length judgment task, obtained from the 16 pattern-matrix trials in the pre-inquiry block and the IA trial in the inquiry block. It was expected that whenever texture segregation occurred, the texture-defined V shape in the background would induce the Ponzo illusion, and would make 
A

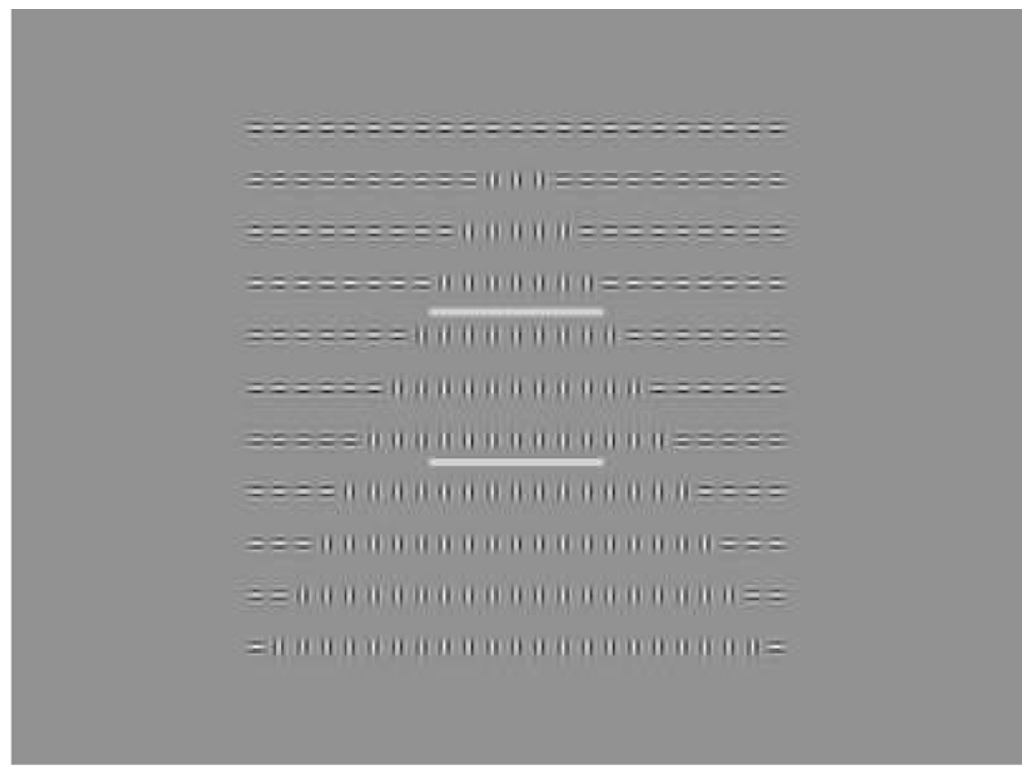

B

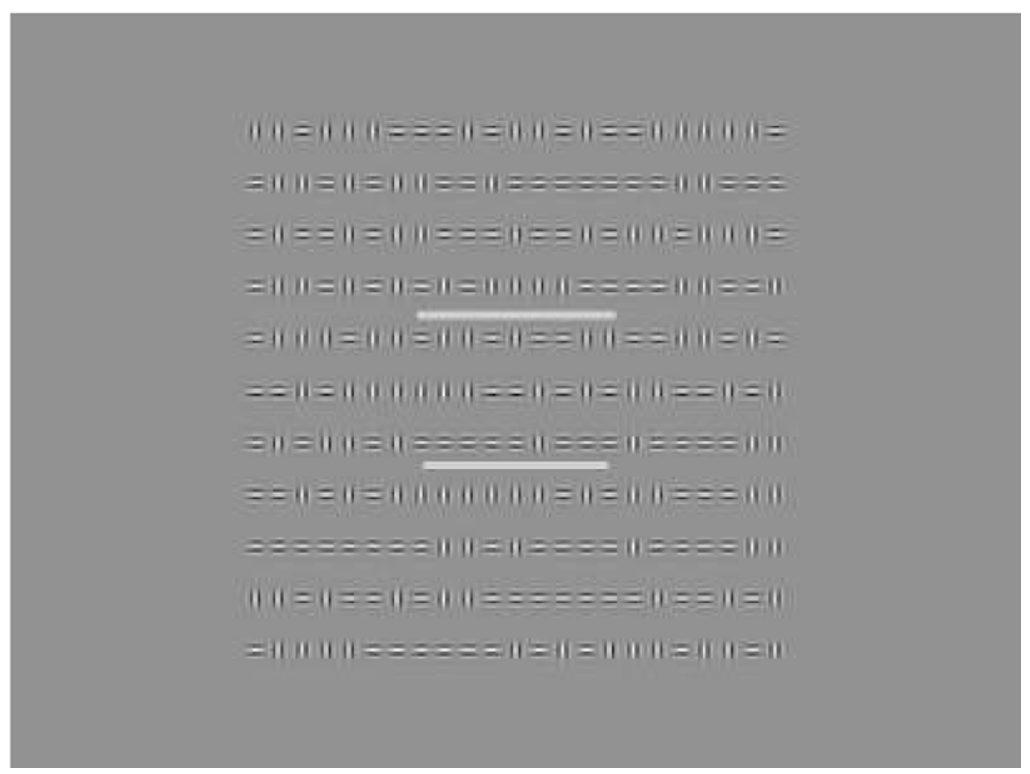

Fig. 2. Examples of the test displays in (A) the pattern-matrix trial and (B) the random-matrix trial in Experiment 1.

the line at the converging end of the $\mathrm{V}$ shape appear longer. The percentage of line-length judgments (Fig. 4) in accordance with the Ponzo illusion was $52 \%$ in the 200 -ms session, which was not significantly higher than chance $(t(42)=0.77, p>.05$, $d=0.12$ ). However, it was $62 \%$ in the 500 -ms session, significantly higher than chance $(t(40)=4.53, p<.01, d=0.71)$.

Thus, the performance was affected by the background only in the 500-ms session. In other words, implicit texture segregation occurs when the duration of exposure of the target display is $500 \mathrm{~ms}$ but not when it is $200 \mathrm{~ms}$. The negative result in the 200-ms session was not due to an inability to conduct the line-length judgment, because the accuracy for randommatrix trials, in which the two lines indeed differed in length, was $96 \%$.

Finally, confidence ratings differed for IA, DA, and FA trials, in the $200-\mathrm{ms}$ session $(1.35,1.60$, and 2.67 , respectively; $F(2,84)=49.42, p<.0001, f=0.88)$ and the 500 -ms session $(1.39,1.80$, and 2.90 , respectively; $F(2,80)=62.71, p<.0001$, $f=1$ ). In the 200-ms session, Tukey's test showed significant differences between the confident ratings in the IA and FA trials, and between the DA and FA trials. In the 500-ms session, Tukey's test showed significant differences for each pair of comparisons among IA, DA, and FA trials. These confidence ratings reflect the participants' subjective feelings of having seen the background, and the results indicated that their subjective feeling of having seen the background correlated with whether the participant had been aware of it. 


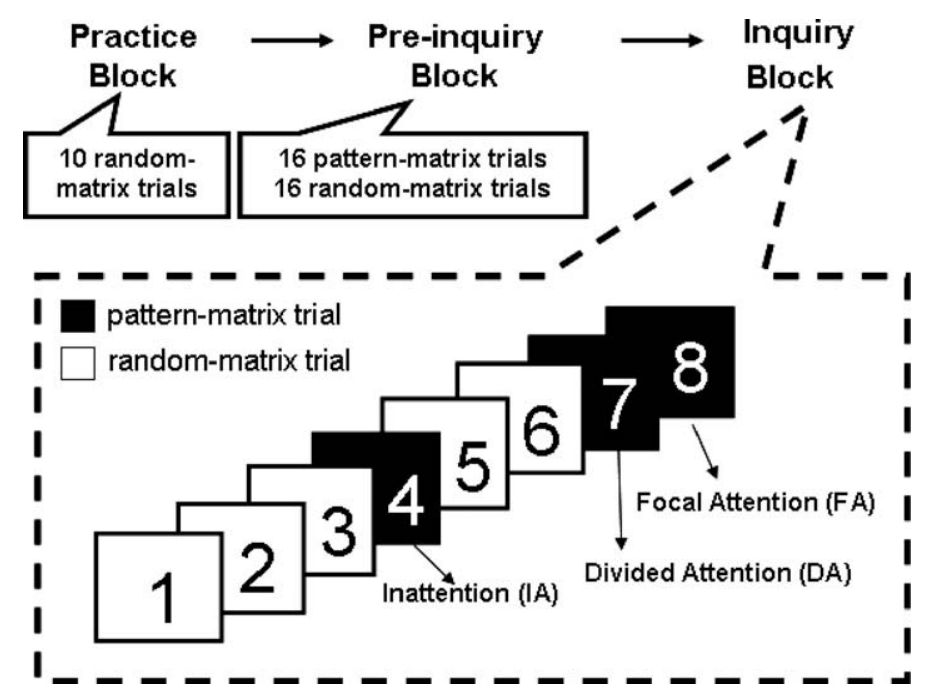

Fig. 3. Procedure in Experiment 1.

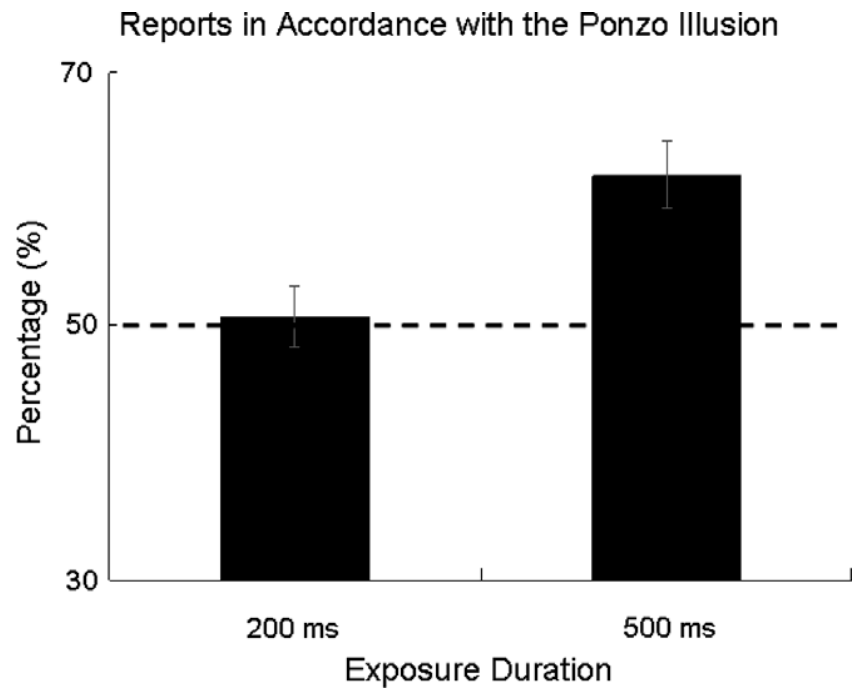

Fig. 4. Percentages of reports of the line-length judgment task in accordance with the Ponzo illusion in Experiment 1.

\section{Experiment 2}

If processing time and awareness can be dissociated, there should be another process that requires awareness but not sufficient processing time, in contrast with the texture segregation found in Experiment 1, which requires sufficient processing time but not awareness. The Simon effect, which refers to the phenomenon that task-irrelevant spatial information influences the response (e.g., Kerzel, Hommel, \& Bekkering, 2001; Simon \& Rudell, 1967; Valle-Inclan, Hackley, \& de Labra, 2002), could be a candidate for this, based on previous finding that the Simon effect requires awareness (Moore et al., 2004). To be comparable with Experiment 1, the exposure duration of the critical stimulus was also kept to 200 and 500 ms, a betweensubject factor.

\subsection{Participants}

Two groups of participants as described before were tested ( $N=26$ in the $200-\mathrm{ms}$ exposure session and $N=29$ in the 500 ms exposure session). None had participated in previous IB experiments before. 


\subsection{Stimuli and design}

Four displays were presented in each trial: the fixation, placeholder, target, and mask displays (Fig. 5). The fixation display contained a central black dot with a diameter of $0.2^{\circ}$ on a homogeneous gray background, followed by the placeholder and the target at the same location. The placeholder was a black numeral " 8 " $\left(0.6^{\circ} \times 0.4^{\circ}\right)$ superimposed on a background pattern consisting of 253 Gabors presented as in Experiment 1, except for their orientations. Among these Gabors, three vertical ones embedded among horizontal ones served as a distracter, and they appeared either to the right or to the left of the numeral " 8 ", at a $1.5^{\circ}$ peripheral location. The target was either a letter " $\mathrm{H}$ " or " $\mathrm{S}$ ", formed by removing two segments from the numeral "8". The mask was identical to that used in Experiment 1.

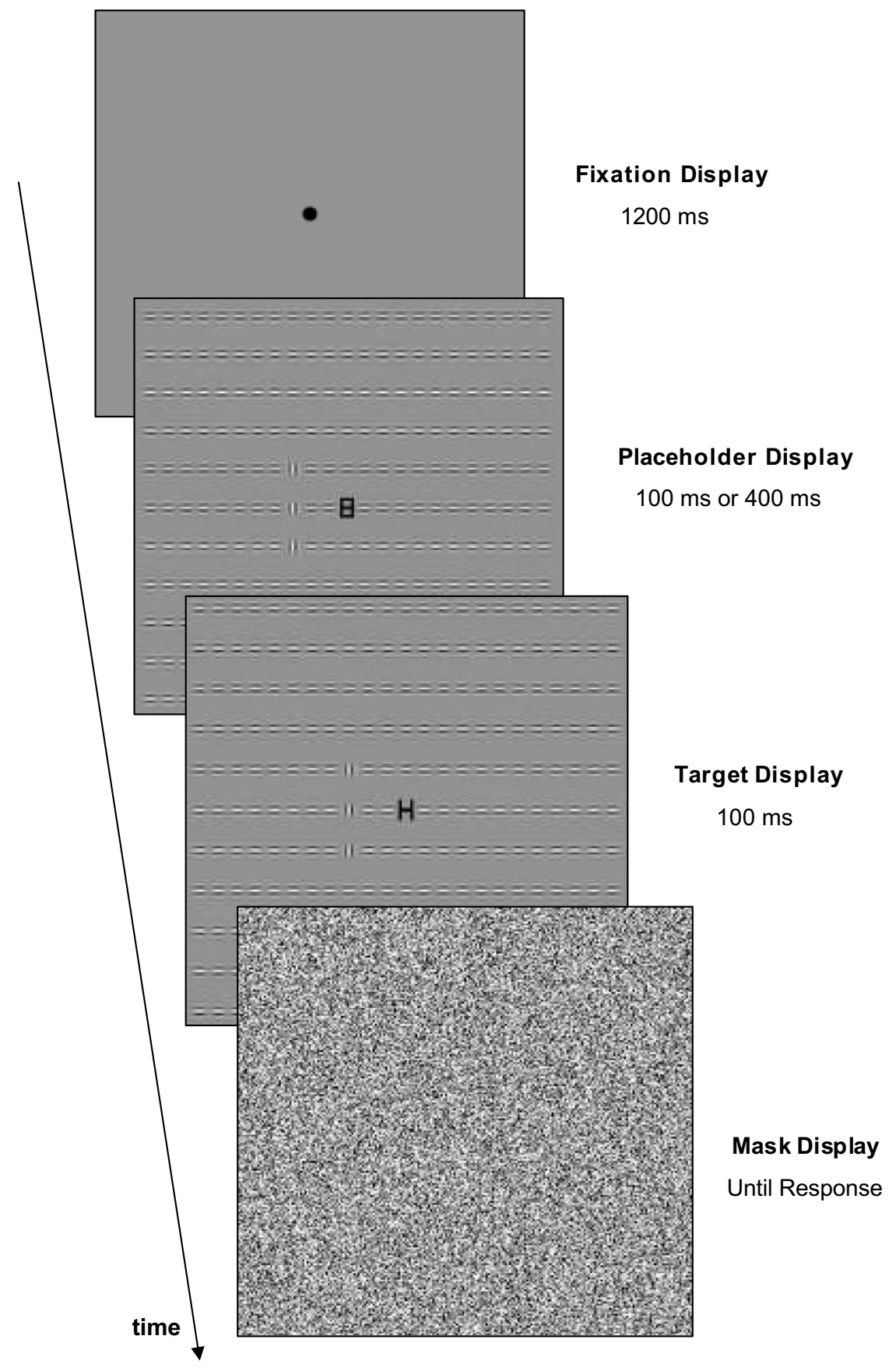

Fig. 5. Stimuli presented in a single trial in Experiment 2. Three vertical Gabor patches served as a distracter appearing either to the right side or the left side of the fixation. 
Each trial began with a fixation dot (1200 ms), followed by the placeholder (100 or $400 \mathrm{~ms}$ ), and then the target (100 ms). The mask appeared on the screen until the participants responded. The distracter appeared simultaneously with the placeholder, and remained on the screen until the mask replaced the target. Thus, the total exposure duration of the distracter was 200 or $500 \mathrm{~ms}$, parallel to that of the texture-defined background pattern in Experiment 1.

\subsection{Procedure}

The participants were asked to discriminate the letter in the target display, by pressing ' $z$ ' with the left index finger if the target was ' $\mathrm{H}$ ' and '/' with the right index finger if ' $\mathrm{S}$ '. If they responded incorrectly, an $800 \mathrm{~Hz}$ tone would be presented as feedback for $200 \mathrm{~ms}$. The location of the distracter was manipulated in order to vary the compatibility between the stimulus and the response. If the location of the distracter and that of the response key were at the same side, this trial was a compatible one; otherwise it was an incompatible one.

An experimental session consisted of four blocks: the practice, pre-inquiry, inquiry, and post-inquiry blocks (Fig. 6). The practice block contained 20 filler trials, in which all items in the background were horizontal Gabors so that there was no distracter. The pre-inquiry block contained 65 trials; the first one was a filler trial, and subsequent 64 trials were comprised half of compatible and half of incompatible trials, presented in a random order.

The inquiry block consisted of nine trials, and the distracter was only presented in the 4th (IA), 8th (DA), and 9th (FA) trials. The location of the distracter in the IA trials was counter-balanced between participants, so that half of the participants saw the left distracter and half of them saw the right one. In the IA and DA trials, in addition to the discrimination task, the participants were also asked to answer three questions, two being identical to those in Experiment 1 . The only different question was the forced-choice one: "If there were three different patches in the background, were they at the left or the right side?" While asking this question, the experimenter presented a card, on which a placeholder and three vertical lines were drawn at the left and right sides for the participants to point to. In the final (FA) trial, the participants were asked to attend only to the background, answer the forced-choice question, and rate their confidence just like those in the IA and DA trials. They were then asked: "Before the first question about the background, did you see any pattern in this background?" Similar to Experiment 1, a positive answer to this question would lead to the exclusion of the participant's data.

After the inquiry block, the participants were required to do a letter discrimination task again in the following post-inquiry block. The stimuli and design of this post-inquiry block were identical to those in the pre-inquiry block, for a fair comparison between results from the two. We included this block in Experiment 2 but not in Experiment 1, because in Experiment 1, the FA trial could be an indicator of whether the participants were able to perceive the stimuli. This was important because if a null result was obtained from the pre-inquiry block, it might be due to the invisibility of the critical stimuli rather than a lack of awareness, and the performance in the FA trials could indicate whether this is the case. However, the Simon effect cannot be tested using a single trial, and thus a larger number of trials, such as those used in the post-inquiry block, are needed.

\subsection{Results}

Data from three participants in the 200 -ms session, and four participants in the 500 -ms session, were excluded due to their reporting having seen the distracter in the pre-inquiry block. The accuracy as to whether the distracter was at the right or the left side, in the IA, DA, and FA trials, respectively, was $47.83 \%\left(\chi^{2}=0.04, p>.05\right), 86.96 \%\left(\chi^{2}=12.57, p<.05\right)$, and $100 \%$

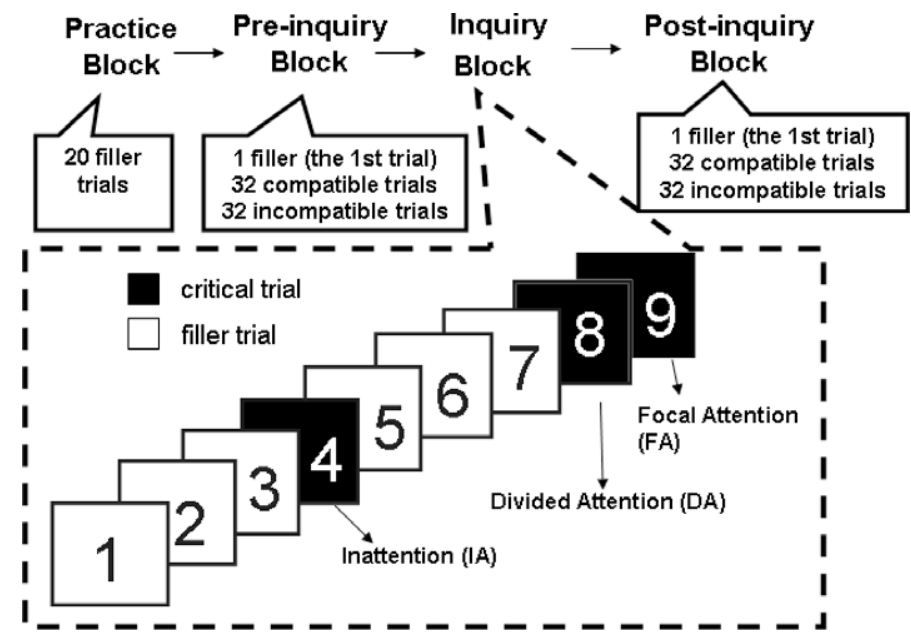

Fig. 6. Procedure in Experiment 2. 
$\left(\chi^{2}=23, p<.05\right)$ in the $200-\mathrm{ms}$ session, and $44 \%\left(\chi^{2}=0.36, p>.05\right), 70 \%\left(\chi^{2}=3.24, p>.05\right)$, and $100 \%\left(\chi^{2}=25, p<.05\right)$ in the 500 -ms session. The accuracy as to the location of the distracter in the IA trials in both 200-ms and 500-ms sessions was not significantly higher than chance-level, confirming that the participants were unaware of the distracter in the pre-inquiry block. The reaction time (RT) data are shown in Fig. 7. There was no reliable Simon effect in the pre-inquiry block. The mean RT differences between compatible and incompatible trials in the pre-inquiry block in the 200- and 500-ms session were $5.51 \mathrm{~ms}(t(22)=1.73, p>.05, d=0.36)$ and $0.03 \mathrm{~ms}(t(24)=0.01, p>.05, d=0.002)$; both did not reach the significant level. However, the Simon effect was observed in the post-inquiry block, with a significant mean RT differences of 12.94 ms $(t(22)=3.79, p<.01, d=0.79)$ in the 200 -ms session, and $14.95 \mathrm{~ms}(t(24)=4.21, p<.01, d=0.84)$ in the $500-\mathrm{ms}$ session. The mean accuracy data yielded similar results. The mean accuracy differences between the compatible and incompatible trials in the pre-inquiry block were $0.1 \%(t(22)=0.1, p>.05, d=0.02)$ in the 200 -ms session and $0.5 \%(t(24)=0.61, p>.05$, $d=0.12$ ) in the 500 -ms session: neither reached a significant level. However, significant accuracy differences of $2.0 \%$ $(t(22)=2.09, p<.05, d=0.44)$ in the 200 -ms session and $3.5 \%(t(24)=3.18, p<.01, d=0.64)$ in the 500 -ms session were observed.

The confidence ratings differed across different types of trials in both 200 -ms $(F(2,44)=62.71, p<.0001, f=1)$ and 500 ms sessions $(F(2,48)=43.83, p<.0001, f=1)$. The mean confidence ratings were $1.22,2.17$, and 2.83 for the IA, DA, and FA trials in the $200-\mathrm{ms}$ session and $1.44,2.12$, and 3.00 in the $500-\mathrm{ms}$ session. In both sessions, Tukey's test showed significant differences between each pair of the three types of trials, again indicating that the participant's subjective feeling of knowing correlated with whether the participant had been aware of the background pattern.

\section{General discussion}

We have shown that texture segregation does not require awareness, provided sufficient available processing time, whereas the Simon effect requires awareness regardless of processing time. These findings reveal the dissociation between processing time and awareness, and do not support single-stream visual processing theories.
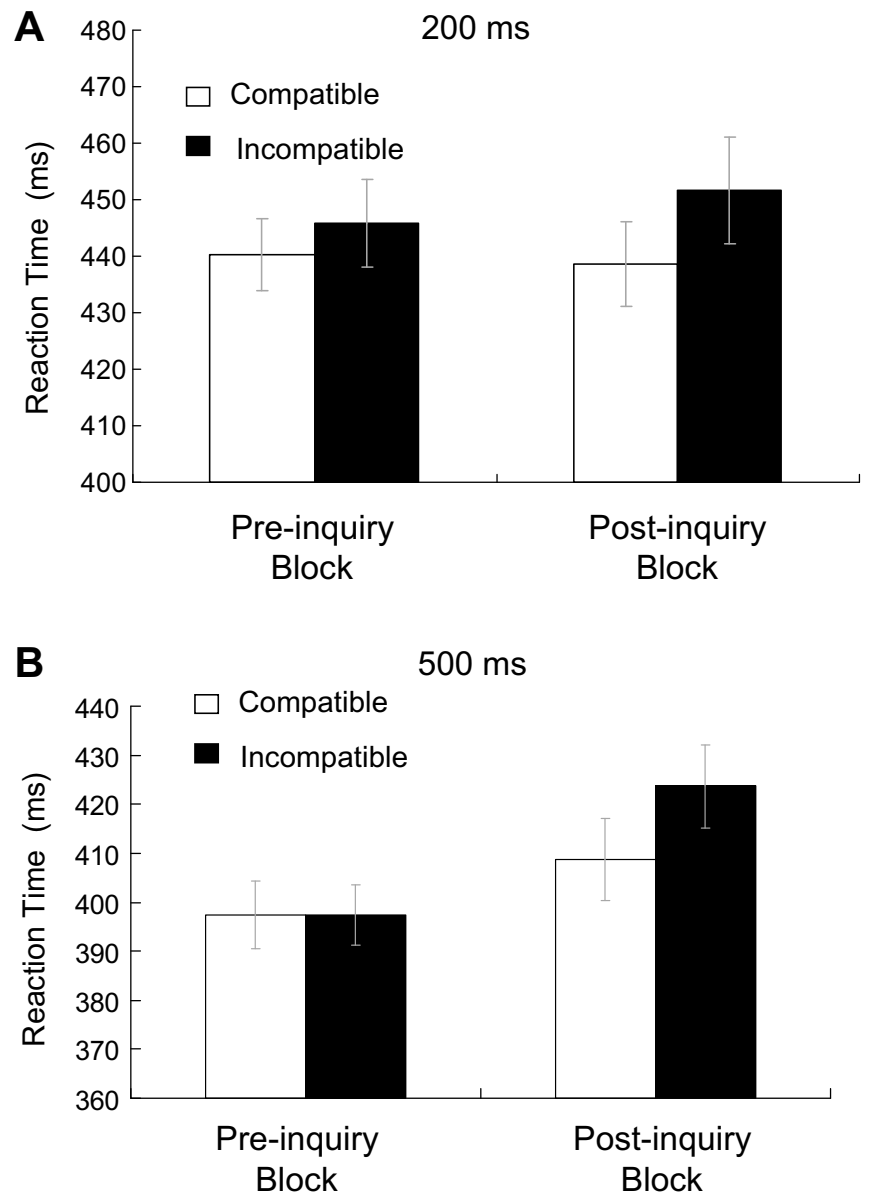

Fig. 7. Mean reaction times for the letter discrimination task in the (A) 200-ms session and (B) 500-ms session in Experiment 2. 
The results of Experiment 1 are consistent with previous studies showing that texture segregation does not require attention (Beck, 1972; Beck, Prazdny, \& Rosenfeld, 1983; Julesz, 1981; Neisser, 1967; Treisman, 1985) and inconsistent with Mack and Rock (1998) showing that texture segregation requires attention. This discrepancy may be due to the insensitivity of the kind of IB task Mack and Rock (1998) used. In the present study, we used an online version of the IB paradigm and showed that texture segregation can indeed occur without awareness. Awareness and attention may be two different psychological entities, but as stated before, if we consider attention as a gate to visual awareness (Dehaene \& Naccache, 2001), a pre-attentive process should also occur without awareness.

One may argue that texture segregation did occur, in Experiment 1, in the 200-ms exposure duration session, but the exposure was too short for the texture-defined $\mathrm{V}$ shape to affect the line-length judgment performance. The results in Moore and Egeth (1997) and Yeh, Chang, Chen, and Li (2001) may hint at the answer to this question. In Moore and Egeth (1997) study, the Ponzo illusion, induced by similarity-grouped background, biased the performance with the exposure duration of $200 \mathrm{~ms}$, so as Yeh et al's. (2001) study with a collinearity-grouped background consisting of Gabors as used here. Based on these results, the exposure duration of $200 \mathrm{~ms}$ should be sufficient for the Ponzo illusion to exert its effect, and the absence of this effect should be attributed to the absence of texture segregation.

One might also argue that with a longer exposure the Simon effect might arise-that 500 ms was still too short a duration. We do not think this is the case. Compared to the durations used in other studies of the Simon effect, 500 ms is a relatively long exposure time. In fact, past studies have shown that the Simon effect decays over time (Hommel, 1993, 1994), and thus it is expected that an exposure duration longer than $500 \mathrm{~ms}$ should only hamper rather than enhance it. Alternatively, could it instead be that the duration of $500 \mathrm{~ms}$ was too long? We also had a 200-ms session, in which no Simon effect was found. As we have confirmed the validity of our experiment by demonstrating the occurrence of the Simon effect in the post-inquiry block, the lack of Simon effect in the pre-inquiry block of Experiment 2 is more likely to be due to the absence of awareness, rather than the exposure time. Is it possible that the Simon effect can occur in two ways, one with awareness, such as in our post-inquiry blocks of Experiment 2, and the other one without awareness, as long as an appropriate time is provided as it was for texture segregation? Given that we have not exhausted all possible exposure durations, this possibility cannot be excluded. However, it is not favored under the Occam's razor. Further studies need to systematically manipulate the time course and provide empirical tests to tease these possibilities apart. For our present purpose, at least under the comparable conditions, the Simon effect and texture segregation are processed differently, in terms of their relationships to awareness.

Processing time is an important factor in visual cognition (e.g., Posner \& Cohen, 1984; Rauschenberger \& Yantis, 2001). However, the time course of implicit processing is rarely examined due to an assumption that a long processing time involves top-down factors such as awareness and attention. Based on this assumption, studies examining unattended processes of clearly visible stimuli often use a very brief exposure duration (e.g., Braun \& Sagi, 1990; Julesz, 1981). However, this dismisses the existence of a process that can occur without awareness but requires time, like the texture segregation shown in this study. Thus, an important message from the current study is that the manipulation of presentation time is necessary for future studies concerning perception without awareness. Ariga, Yokosawa, and Ogawa (2007) study on the same-object advantage (i.e., the RT benefit when the cue and target are on the same-object compared to when they are on different objects, Egly, Driver, \& Rafal, 1994) could be an example. They used the IB paradigm and found that when the observers were unaware of the illusory object, the same-object advantage was absent. In their study, the object was presented simultaneously with the target, thus the exposure duration of the object co-varied with the reaction time. Given that available processing time is crucial for the occurrence of an effect without awareness, as implicated in this study, the absence of the same-object advantage in Ariga et al's. (2007) study might be due to insufficient processing time rather than a lack of awareness on the visual objects.

Recently, there has been a growing number of studies showing that object recognition can occur without awareness. For example, Mitroff and Scholl (2005) used the MIB paradigm and found that forming and updating of object representation does not require awareness; nor does surface completion (perceiving an illusory surface which is occluding or occluded by another object) using the IB paradigm in Moore et al. (2003). Given these findings, it may not be surprising that texture segregation occurs without awareness. Nevertheless, unlike previous studies demonstrating the level at which implicit processing can occur, we stress the influence of processing time, which is usually presumed to co-vary with awareness.

Contrary to the view that processing time and awareness are interdependent, our findings suggest their independence from each other, and can be explained in the following way. When the participant is conducting a certain task, two streams of information are ongoing. The explicit stream deals with the main task, such as the line-length judgment task in Experiment 1 or the letter discrimination task in Experiment 2; whereas the implicit stream deals with the background patterns, such as the texture-defined $\mathrm{V}$ shape in Experiment 1 or the distracter in Experiment 2. When the information is still in the implicit stream, some process like texture segregation is taking place, and available processing time is important. However, information in this stream neither provides the participants their feeling of knowing nor induces the stimulus-response compatibility effect, leading to both the participants' inability to report and the absence of the Simon effect. Such a notion can also be framed within the neuronal global workspace model of consciousness (Dehaene \& Naccache, 2001). According to this model, the mechanism of texture segregation can be viewed as a module that operates without awareness, and thus the performance of the line-length judgment is influenced by the texture background even though the participants are unaware of the $\mathrm{V}$ shape in the background. Compared to a perceptual effect such as texture segregation, the Simon effect, which is considered to be due to response-selection (Kornblum, Stevens, Whipple, \& Requin, 1999; Simon, Acosta, Mewaldt, \& Speidel, 1976), requires the involvement of the global workspace, and thus cannot occur without awareness. 
As mentioned earlier, many methods have been developed to probe perception without awareness. A major constraint of these methods is the difficulty in controlling the presentation time, including the need for brief exposure duration in the attentional blink or repetition blindness paradigms, or the involuntary oscillation between two perceptual states in the bistable figures or MIB/PFI paradigms. A newly developed method, the continuous flash suppression (CFS), may avoid this oscillation problem thus it can be employed to test the time course of a process without awareness. In this method, one eye is presented with the critical stimulus and the other with high-contrast flashing Mondrians, and the dominance of the Mondrians in binocular rivalry renders the critical stimulus invisible (Jiang, Costello, Fang, Huang, \& He, 2006). This method has much potential as to the issue of what can be processed without awareness. However, a very long exposure time, usually more than one second, is required for the suppressed stimulus to exert its effect (e.g., Jiang, Costello, \& He, 2007; Jiang \& He, 2006; Tsuchiya \& Koch, 2005). The necessity for long exposure in this method leads to coarse temporal resolution. Compared to CFS, the IB paradigm is better suited to capture the time course of a process without awareness.

\section{Conclusion}

Consciousness is an intriguing issue that brings interests of researchers from multiple disciplines, and they have developed many methods to reveal the mystery of consciousness. However, due to difficulties in manipulating the duration of exposure, and the assumption of interdependence between processing time and awareness, the effect of processing time has rarely been systematically examined. We have, by using the IB paradigm, independently manipulated awareness and processing time, and the different result patterns found between texture segregation and the Simon effect reveal how awareness and processing time can be dissociated, which is consistent with the existence of separate streams for implicit and explicit processes.

\section{References}

Ariga, A., Yokosawa, K., \& Ogawa, H. (2007). Object-based attentional selection and awareness of objects. Visual Cognition, 15, 685-709.

Beck, J. (1972). Similarity grouping and peripheral discriminability under uncertainty. American Journal of Psychology, 85, 1-19.

Beck, J., Prazdny, K., \& Rosenfeld, A. (1983). A theory of textural segmentation. In J. Beck, B. Hope, \& A. Rosenfeld (Eds.), Human and machine vision (pp. 1-38). New York: Academic Press.

Bonneh, Y. S., Cooperman, A., \& Sagi, D. (2001). Motion-induced blindness in normal observers. Nature, 411, 798-801.

Braun, J., \& Sagi, D. (1990). Vision outside the focus of attention. Perception E' Psychophysics, 48, 45-58.

Broadbent, D. E. (1958). Perception and Communication. London: Pergamon Press.

Carter, O. L., \& Pettigrew, J. D. (2003). A common oscillator for perceptual rivalries? Perception, 32, $295-305$.

Clarke, F. J. J. (1957). Rapid light adaptation of localised areas of the extra-foveal retina. Optica Acta, 4, 69-77.

Clarke, F. J. J. (1960). A study of Troxler's effect. Optica Acta, 7, 219-236.

Clarke, F. J. J. (1961). Visual recovery following local adaptation of the peripheral retina (Troxler's effect). Optica Acta, 8, 121-135

Clarke, F. J. J., \& Belcher, S. J. (1962). On the localization of Troxler's effect in the visual pathway. Vision Research, 2, 53-68.

Danckert, J., \& Rossetti, Y. (2005). Blindsight in action: What can the different sub-types of blindsight tell us about the control of visually guided actions? Neuroscience E' Biobehavioral Reviews, 29, 1035-1046.

Dehaene, S., \& Naccache, L. (2001). Towards a cognitive neuroscience of consciousness: Basic evidence and a workspace framework. Cognition, $79,1-37$.

Destrebecqz, A., \& Cleeremans, A. (2001). Can sequence learning be implicit? New evidence with the process dissociation procedure. Psychonomic Bulletin and Review, 8, 343-350.

Deutsch, J. A., \& Deutsch, D. (1963). Attention: Some theoretical considerations. Psychological Review, 70, 51-61.

Egly, R., Driver, J., \& Rafal, R. D. (1994). Shifting visual attention between objects and locations: Evidence from normal and parietal lesion subjects. Journal of Experimental Psychology: General, 123, 161-177.

Enns, J. T., \& Di Lollo, V. (1997). Object substitution: A new form of masking in unattended visual locations. Psychological Science, 8, $135-139$.

Faulkner, D., \& Foster, J. K. (2002). The decoupling of “explicit" and "implicit" processing in neuropsychological disorders: Insights into the neural basis of consciousness? Psyche, 8, 1-28.

Graf, E. W., Adams, W. J., \& Lages, M. (2002). Modulating motion-induced blindness with surface completion and depth ordering. Vision Research, 42, 2731-2735.

Hommel, B. (1993). The relationship between stimulus processing and response selection in the Simon task: Evidence for a temporal overlap. Psychological Research/Psychologische Forschung, 55, 280-290.

Hommel, B. (1994). Spontaneous dacay of response-code activation. Psychological Research/Psychologische Forschung, 56, $261-268$.

Hsu, L. C., Yeh, S. L., \& Kramer, P. (2004). Linking motion induced blindness to perceptual filling-in. Vision Research, 44, 2857-2866.

Hsu, L. C., Yeh, S. L., \& Kramer, P. (2006). A common mechanism for perceptual filling-in and motion-induced blindness. Vision Research, 46, $1973-1981$.

Jiang, Y., Costello, P., Fang, F., Huang, M., \& He, S. (2006). A gender- and sexual orientation-dependent spatial attentional effect of invisible images. Proceedings of the National Academy of Sciences of the United States of America, 103, 17048-17052.

Jiang, Y., Costello, P., \& He, S. (2007). Processing of invisible stimuli: Advantage of upright faces and recognizable words in overcoming interocular suppression. Psychological Science, 18, 349-355.

Jiang, Y., \& He, S. (2006). Cortical responses to invisible faces: Dissociating subsystems for facial-information processing. Current Biology, 16, $2023-2029$.

Julesz, B. (1981). Textons, the elements of texture perception, and their interactions. Nature, 290, 91-97.

Kanwisher, N. G. (1987). Repetition blindness: Type recognition without token individuation. Cognition, 27, $117-143$.

Kerzel, D., Hommel, B., \& Bekkering, H. (2001). A Simon effect induced by induced motion and location: Evidence for a direct linkage of cognitive and motor maps. Perception E' Psychophysics, 63, 862-874.

Kim, C.-Y., \& Blake, R. (2005). Psychophysical magic: Rendering the visible 'invisible'. Trends in Cognitive Sciences, 9, $381-388$.

Kornblum, S., Stevens, G. T., Whipple, A., \& Requin, J. (1999). The effects of irrelevant stimuli: 1 . The time course of stimulus-stimulus and stimulusresponse consistency effects with Stroop-like stimuli, Simon-like tasks, and their factorial combinations. Journal of Experimental Psychology: Human Perception $\mathcal{E}$ ' Performance, 25, 688-714.

Mack, A., \& Rock, I. (1998). Inattentional blindness. Cambridge, MA: MIT Press.

Mack, A., Tang, B., Tuma, R., Kahn, S., \& Rock, I. (1992). Perceptual organization and attention. Cognitive Psychology, $24,475-501$.

Milner, A. D., \& Goodale, M. A. (1995). The visual brain in action. New York, NY: Oxford University Press.

Mitroff, S. R., \& Scholl, B. J. (2005). Forming and updating object representations without awareness: Evidence from motion-induced blindness. Vision Research, 45, 961-967. 
Montaser-Kouhsari, L., Moradi, F., Zandvakili, A., \& Esteky, H. (2004). Orientation-selective adaptation during motion-induced blindness. Perception, 33, $249-254$.

Moore, C. M., \& Egeth, H. (1997). Perception without attention: Evidence of grouping under conditions of inattention. Journal of Experimental Psychology: Human Perception and Performance, 23, 339-352.

Moore, C. M., Grosjean, M., \& Lleras, A. (2003). Using inattentional blindness as an operational definition of unattended: The case of surface completion. Visual Cognition, 10, 299-318.

Moore, C. M., Lleras, A., Grosjean, M., \& Marrara, M. T. (2004). Using inattentional blindness as an operational definition of unattended: The case of a respond-end effect. Visual Cognition, 11, 705-719.

Neisser, U. (1967). Cognitive Psychology. New York: Appleton-Century-Crofts.

Posner, M. I., \& Cohen, Y. (1984). Components of visual orienting. In H. Bouma \& D. G. Bouwhuis (Eds.), Attention and performance X: Control of language processes (pp. 531-556). Hillsdale, NJ: Erlbaum.

Ramachandran, V. S., \& Gregory, R. L. (1991). Perceptual filling-in of artificially induced scotomas in human vision. Nature, 350, 699-702.

Ramachandran, V. S., Gregory, R. L., \& Aiken, W. (1993). Perceptual fading of visual texture borders. Vision Research, 33, 717-721.

Rauschenberger, R., \& Yantis, S. (2001). Masking unveils pre-amodal completion representation in visual search. Nature, 410, 369-372.

Raymond, J. E., Shapiro, K. L., \& Arnell, K. M. (1992). Temporary suppression of visual processing in an RSVP task: An attentional blink? Journal of Experimental Psychology: Human Perception and Performance, 18, 849-860.

Rock, I., Linnet, C. M., Grant, P., \& Mack, A. (1992). Perception without attention: Results of a new method. Cognitive Psychology, 24, $502-534$.

Rubin, E. (1921). Visuell wahrgenommene Figuren. Kopenhagen: Glydenaske boghandel.

Simon, J. R., Acosta, E., Mewaldt, S. P., \& Speidel, C. R. (1976). The effect of an irrelevant directional cue on choice reaction time: Duration of the phenomenon and its relation to stages of processing. Perception \& Psychophysics, 19, 16-22.

Simon, J. R., \& Rudell, A. P. (1967). Auditory S-R compatibility: The effect of an irrelevant cue on information processing. Journal of Applied Psychology, 51, 300-304.

Spillmann, L., \& Kurtenbach, A. (1992). Dynamic noise backgrounds facilitate target fading. Vision Research, 32, 1941-1946.

Stoerig, P., \& Cowey, A. (1997). Blindsight in man and monkey. Brain, 120, 535-559.

Treisman, A. M. (1985). Preattentive processing in vision. Computer Vision, Graphics and Image Processing, 31, 156-177.

Troxler, I. P. V. (1804). Über das Verschwinden gegebener Gegenstände innerhalb unseres Gesichtskreises. In J. Himly \& J. A. Schmidt (Eds.). Ophthalmologische Bibliothek (Vol. 2, pp. 1-119). Jena: Fromann.

Tsuchiya, N., \& Koch, C. (2005). Continuous flash suppression reduces negative afterimages. Nature Neuroscience, 8, 1096-1101.

Ungerleider, L. G., \& Mishkin, M. (1982). Two cortical visual systems. In D. J. Ingle, M. A. Goodale, \& R. J. Mansfield (Eds.), Analysis of visual behavior (pp. 549-580). Cambridge, MA: MIT Press.

Valle-Inclan, F., Hackley, S. A., \& de Labra, C. (2002). Does stimulus-driven response activation underlie the Simon effect? In W. Prinz \& B. Hommel (Eds.). Attention and performance: XIX Common mechanisms in perception and action (pp. 474-493). Oxford, UK: Oxford University Press.

Wade, N. J., \& Wenderoth, P. (1978). The influence of colour and contour rivalry on the magnitude of the tilt after-effect. Vision Research, 18, 827-835.

Weiskrantz, L. (1986). Blindsight: A case study and implications. Oxford: Oxford University Press.

Welchman, A. E., \& Harris, J. M. (2000). The effects of dot density and motion coherence on perceptual fading of a target in noise. Spatial Vision, 14, 45-58. Welchman, A. E., \& Harris, J. M. (2001). Filling-in the details on perceptual fading. Vision Research, 41, 2107-2117.

White, K. D., Petry, H. M., Riggs, L. A., \& Miller, J. (1978). Binocular interactions during establishment of McCollough effects. Vision Research, 18, 1201-1215 Wolfe, J. M. (1999). Inattentional amnesia. In V. Coltheart (Ed.), Fleeting memories: Cognition of brief visual stimuli (pp. 71-94). Cambridge, MA: MIT Press. Yeh, S. L., Chang, H. C., Chen, I., \& Li, J. L. (2001). Perceptual organisation with and without attention. Perception, 30(Suppl.), 45a. 